\title{
Haar Discrete Wavelet-Based and Scanning Window for Vehicle License Plate Recognition
}

\author{
Rong-Choi Lee*, King-Chu Hung \\ * Corresponding author. Tel.: +886-973196786; email: u9715902@nkfust.edu.tw \\ Manuscript submitted April 1, 2017; accepted August 12, 2017. \\ doi: 10.17706/jsw.12.8.631-642
}

Department Computer and Communication, Institute of Engineering Science and Technology, College of Engineering, National Kaohsiung First University of Science and Technology, Kaohsiung, Taiwan, R. O. C.

\begin{abstract}
This paper is the study of vehicle license plate recognition. We use the scan window, 2-D Haar Discrete Wavelet Transform and Artificial Neural Networks. This paper consists of three main parts. First part is to locate and extract license plate. Second part is the Artificial Neural Network training of the license plate. Third part is a real time scans recognition. We use two times Haar Discrete Wavelet Transform image, and extract low frequency part LL of vehicles license plate image, makes image coefficient became original of one-sixteen, thus reducing image coefficients, so can to fast recognition.

This method is to use scan the license plate recognition, but we do not license plate recognition for each character. Experiment result can be a high recognition rate.
\end{abstract}

Keywords: Vehicle license plate recognition, discrete wavelet transform, artificial neural network.

\section{Introduction}

\subsection{The Motive of the Research}

The community has experienced continuous development and growth, and today almost all steam locomotives travel, the number of cars and thus rapid surge vehicles; parking, arising from all aspects of vehicle management gradually watched by everyone. Management of traditional the parking lot, mostly to hire someone out of control vehicle in the parking lot entrance fees and the prevention of vehicle theft. This work is quite troublesome for managers, and the vehicles are not always out, and therefore a considerable waste of human resources. In order to improve the traditional management model of the car park need to improve parking management mechanism, it is automated, digital management, effective and accurate control for all vehicles.

In order to save manpower needs, the use of license plate recognition function to achieve its objectives, so have a license plate recognition system (License-Plate Recognition, LPR) appear, automated license plate recognition systems can assist the human can even replace the surplus of human resources in vehicle management, as long as the gate at placement of a license plate recognition systems can identify whether a vehicle to enter, and to test whether the vehicle is oriented building permit to enter the vehicle, and then determine whether to be passed, and so can help management to facilitate control into and out of vehicles.

Most of the traditional license plate recognition systems, the main process before such as image processing, license plate location, character cut, character recognition steps. License plate recognition system can be applied to the environment can be divided into three spaces:

(1) Applied to the open space, refers to taking the application of conditions loose and database unlimited number of vehicles; such as highways, roadside parking space, and so on.

(2) Applied to non-open space, refers to a limited area and limited database application environment; such as community parking and pay parking, automated access control and detection, and so on. 
(3) Applies to two kinds of space, defined in the applicable above place algorithms, such as: highways, community parking and pay parking lots, and so on. Especially open space and applies to two kinds of space technology, challenging the highest outdoor vulnerable to weather changes or the influence of human factors during the day, night, sunny, rainy, weather factors will affect the light in brightness variations on the license plate, in addition to the background of the image is also easy to be ready to change, and changes in brightness as well as complex background often leads to the recognition of the difficulties; the contrary, non-open space is less susceptible to weather The influence of light on a license plate change and the background of the image is fixed in such a simple environment, research and development is relatively easy.

The purpose of this paper is set to automatic license plate recognition system used in the "open space". We have to find a hundred vehicles to carry out the experiment, each vehicle are six kinds of distance sampling, simulation of a road between the vehicles, and all samples to build the database.

This system replaces the traditional license plate recognition system image pre-processing, license plate location and other steps, the use of a scanning window, perform a one-dimensional non-recursive discrete periodic wavelet transform to synchronize the scanning process, combined with back-propagation neural network (Back Propagation Neural Network) technology to create a license plate recognition system architecture, and hope to find the best neural network parameters to improve the overall recognition rate, and eventually through the license plate recognition than by way of the wavelet coefficients to verify correct.

To suggest the thesis that is because of the promotion of life quality, vehicles have become an essential vehicle. The increase of vehicle number produces some management problems, such as the building vehicles, company vehicles management. Most of the current vehicle is use of human resources management. Therefore, we propose a applications in a limited area of automatic license plate recognition system to control the vehicles, so can save on labor costs and improve efficiency.

Into the principal purpose of this research program who want to effective recognition of the license plates of moving vehicles, and license plate recognition can improve the success rate, and hope that through our research, can learn about the hardware on the feasibility of, and can provide wish to engage in research in this area and some reference direction.

\subsection{Research of Related Literature}

In [1-4] are real-time license plate recognition, and in [5-8] are make a lot of ways the license plate location. In [1] use HSI color space method. In $[2-4,13,20]$ are the license-plate characters recognition, but the similar characters confusion problem, such as 1 and I, 0 and $0, \mathrm{~S}$ and 5, and so on. In [5] need to create a reference model, and the standard license plate for recognition, but the position photography is different, so its easily lead to recognition errors. In [6] only make the location of the vehicle's license plate. In [7] that because the image does not at the same position to photography. Hence, they will not be easy for the license plate match. In [8] need to find the thresholds of row and column, but affected by light or night and other factors, it cannot find the ideal thresholds. In [15] use pattern matching for real time detection of license plates, because the image does not at the same position to photography and road has slope. Hence, they will not be effective for the license plate matching.

\subsection{This Thesis Proposed of Method}

This article is in upgrade license plate recognition, and current Taiwan license plate size is $150 \mathrm{~mm} \mathrm{x320} \mathrm{mm}$, vehicle grade by two English letters and four digitals, license plate number word and word interval for $10 \mathrm{~mm}$, lack space and correctly of cutting, to improved missing, we using scan window combination Haar Discrete Wavelet Transform (HDWT) [6] and using artificial neural network [9], [10], [14], [21] engaged in license plate recognition.

This article mainly into training and license plate recognition of vehicle license plate recognition system in two parts, in the first part of training data is to use a vehicle license plate edge functions and Sobel shield operator to 
detect license plate, and automatic search for vehicle license plate location and extract license plate. In the first part of the license plate training, training data is to use a vehicle license plate detection of edge detection and Sobel operator license plates, automatic search for vehicle license plate location and extract license plate. The second part license plate recognition is the use of $32 \times 80$ window to scan, scan window area through two times Haar Discrete Wavelet Transform, each image after the conversion into HH, HL, LH, LL and other four part, where LL is a low frequency images and extract the LL low-frequency parts of the image, and using artificial neural network to a limited area of vehicle license plate recognition. Therefore, this method of vehicle license plate character segmentation problems can be avoided.

In [1]-[4], [15] are real-time license plate recognition, and in [5]-[8], [16] are make a lot of ways the license plate location. In [1] use HSI color space method. In [2]-[4] are the license-plate characters recognition, but the similar characters confusion problem, such as 1 and I, 0 and $0, S$ and 5 , and so on. In [6] only make the location of the vehicle's license plate. In [7] that because the image does not at the same position to photography. Hence, they will not be easy for the license plate match. In [8] need to find the thresholds of row and column, but affected by light or night and other factors, it cannot find the ideal thresholds.

\section{Methods}

After we study the way is to scan the image in this article, use 2D Haar discrete wavelet transform and artificial neural network for recognition. In 2.1 Section is edge function and Sobel mask operator, 2.2 Section is 2D Haar discrete wavelet transform of introduction, 2.3 Section is the introduction of artificial neural network, described later.

\subsection{Edge Function and Sobel Mask Operator}

Edge function and Sobel mask operator as $(1,2)$ formula for license plate localization, it can automatically detect the critical threshold, the following (3) formula is MATLAB software program. Images transform into 0 and 1 binary, making license plate and background distinct.

$$
\text { sobel }=\left[\begin{array}{rrr}
1 & 2 & 1 \\
0 & 0 & 0 \\
-1 & -2 & -1
\end{array}\right]
$$

formula is a horizontal edge operators.

$$
\text { sobel }=\left[\begin{array}{ccc}
-1 & 0 & 1 \\
-2 & 0 & 2 \\
-1 & 0 & 1
\end{array}\right]
$$

formula is vertical edge operators.

$$
\text { [Image1, threshold]=edge (Image, 'sobel') }
$$

The 'Image1' character is after edge image the output image, the 'threshold' character is edge threshold parameter of image, the 'Image' character is input image, the 'sobel' character is sobel mask operator, the 'edge' character is edge function.

\subsection{2-D Haar Discrete Wavelet Transform}

The discrete wavelet transform is a very popular method in digital image processing in recent years, especially in multi-resolution representation. The discrete wavelet transform can decompose an image into some 
sub-bands and encodes the signal of sub-bands according to the importance of signal. discrete wavelet transform turns an image into high frequency and low frequency data. According to these different data, we can do the processing respectively. Due to the sensitivity of human's eyes, the low frequency data forms the important part of the original image. If the values of low frequency coefficients change, one can recognize the change easily. On the other hand, human's eyes are much less sensitive to the high frequency part, because it is hard for a human to discover it.

The 2D Haar discrete wavelet transform [7] can decompose an image into four sub-bands. Have high frequency ( $\mathrm{HH}$ ) and the high and low frequency (HL) and low and high frequency (LH) and low frequency (LL) the four sub-bands. Low frequency (LL) between the pixels of change is relatively small, image clearer, so after each conversion only to extract low frequency (LL) part. Haar transform characteristics of: (a) Multiplication is not required. (b)Input and output points the same. (c) Frequency two parts, low frequency all is 1 and high frequency (half are 1, half are -1 ), as show in from (4) to (6). (d)You can analyze a signal localized features. (e) Instruction cycle very fast. But it does not suitable for signal analysis. Haar transform is one of the easiest and reaction time-variant spectrum image and signal representation Haar transform, Haar transform matrix such as following,

When $N=2$,

$$
\mathbf{H}=\left[\begin{array}{cc}
1 & 1 \\
1 & -1
\end{array}\right]
$$

When $\mathrm{N}=4$,

$$
\mathbf{H}=\left[\begin{array}{cccc}
1 & 1 & 1 & 1 \\
1 & 1 & -1 & -1 \\
1 & -1 & 0 & 0 \\
0 & 0 & 1 & -1
\end{array}\right]
$$

Inverse Haar Transform property,

$$
\begin{gathered}
\mathrm{H}^{-1}=\mathrm{H}^{\mathrm{T}} \mathrm{D} \\
\mathrm{D}[m, n]=0, \text { if } m \neq n \\
\mathrm{D}[0,0]=2^{-k}, \mathrm{D}[1,1]=2^{-k}, \\
\mathrm{D}[n, n]=2^{-k+p}, \text { if } 2 p \leq n<2 p+1 .
\end{gathered}
$$

The $\mathrm{m}$ is row, $\mathrm{n}$ is column, $\mathrm{k}$ is index number, $\mathrm{p}$ is position integer number.

When $k=3$,

$$
\mathbf{D}=\left[\begin{array}{cccccccc}
1 / 8 & 0 & 0 & 0 & 0 & 0 & 0 & 0 \\
0 & 1 / 8 & 0 & 0 & 0 & 0 & 0 & 0 \\
0 & 0 & 1 / 4 & 0 & 0 & 0 & 0 & 0 \\
0 & 0 & 0 & 1 / 4 & 0 & 0 & 0 & 0 \\
0 & 0 & 0 & 0 & 1 / 2 & 0 & 0 & 0 \\
0 & 0 & 0 & 0 & 0 & 1 / 2 & 0 & 0 \\
0 & 0 & 0 & 0 & 0 & 0 & 1 / 2 & 0 \\
0 & 0 & 0 & 0 & 0 & 0 & 0 & 1 / 2
\end{array}\right]
$$

Discrete Wavelet Transform (DWT) is a novel mathematical tool for digital signal processing, discrete wavelet transform turns an image in spatial domain into an image in frequency domain. The image in frequency domain consists of high frequency and low frequency data. According to these different data, we can do the processing respectively. Due to the sensitivity of human's eyes, the low frequency data forms the important part of the original image. If the values of low frequency coefficients change, one can recognize the change easily. On the 
other hand, human's eyes are much less sensitive to the high frequency coefficients change, it is hard for a human being to discover it. This is the main merit of discrete wavelet transform in the fields of digital signal processing and image compression.

There are many different discrete wavelet transform proposed. In this sub-section, we introduce the simplest one, Haar DWT. For digging the frequency features from the original image, we calculate the DWT coefficients by the addition and subtraction operations. After the addition operation, the pixel value becomes bigger. The bigger the resulted coefficient is, the more important it stands for the image. The results of addition operation are the low frequency part and the results of subtraction operation are the high frequency part.

\subsection{Artificial Neural Network}

Artificial neural Network the network as show in Fig. 1, the network level can be divided into input layer, hidden layer and the output layer and other three. Output transfer function between the layers is to use the hyperbolic tangent function (Tan-sigmoid Transfer function), finally, binary convert to decimal output number value.

The network's hidden layer can acquire (8) formula into the hyperbolic tangent function, (9) formula is the hidden layer of network output. Network output layer can acquire (10) formula into the hyperbolic tangent function, (11) formula the network output is the output layer.

$$
\begin{aligned}
& \text { net }_{h}=\sum_{X=1}^{n} W_{x h} \cdot X_{X}-\theta_{h} \\
& f\left(\text { net }_{h}\right)=\frac{e^{\text {net }} h-e^{- \text {net }_{h}}}{e^{\text {net }} h+e^{- \text {net }_{h}}} \\
& \text { net }_{\mathrm{y}}=\sum_{\mathrm{y}=1}^{\mathrm{h}} \mathrm{W}_{\mathrm{hy}} \cdot \mathrm{f}\left(\text { net }_{\mathrm{h}}\right)-\theta_{\mathrm{y}}
\end{aligned}
$$

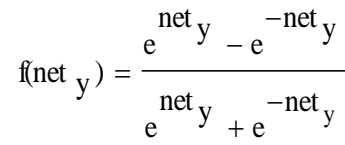

where $X_{x}$ is neural network input neuron, $W_{x h}$ is weight of input layer and hidden layer, net $_{h}$ is sum of hidden layer, $\theta_{h}$ is basis of hidden layer, $f\left(\right.$ net $\left._{h}\right)$ is transfer function of hidden layer, $W_{h y}$ is weight of hidden layer and

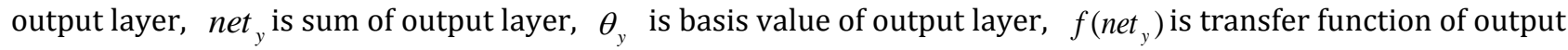
layer, $\mathrm{h}$ is nerve unit number of Hidden layer.

Artificial neural network in this article is the special value output layer neurons converting binary decimal output, and output layer neurons of the components of the target value converted to a decimal value for comparison. Such as output values and target values are the same for training completed, or the express train failed, return train.

\section{Training and Recognition License Plate Steps}

This license plate recognition system is divided into two parts, the first is training, and the second is used $32 \times 80$ resolution windows scan recognition. Training and recognition steps described in below instructions.

\subsection{Simulation Software and Hardware Environment}

Using personal computer simulation, the software is Microsoft Windows XP, home edition, version 2002, 
service pack2, hardware is Compaq Presario CQ2020TW computer, Intel@ ATOM ${ }^{\text {TM }} 230$ processor, $1024 \mathrm{MB}$ DDR2, 160GB hard disk space.

\subsection{Vehicle License Plate Photograph}

Using the CASIO EXILIM, 10.1 mega pixels digital camera EX-S10, first adjust the camera resolution $480 \times 640$, according to Fig. 1 of 12-0 mark for vehicle license plate location to shoot, as the license plate training samples. Fig. 1 12-0 mark location of any 6 locations within the region, shooting 6 vehicle license plates, as license plate recognition samples.

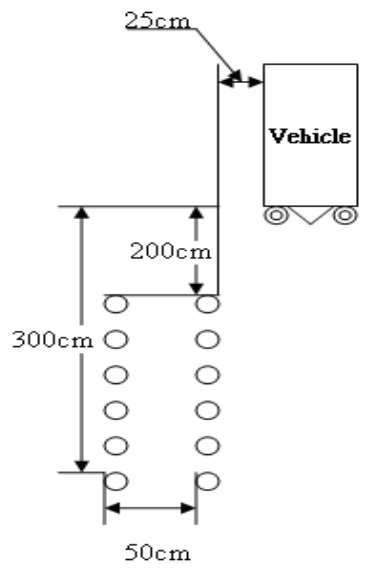

Fig. 1. Vehicle image photo shoot location map.

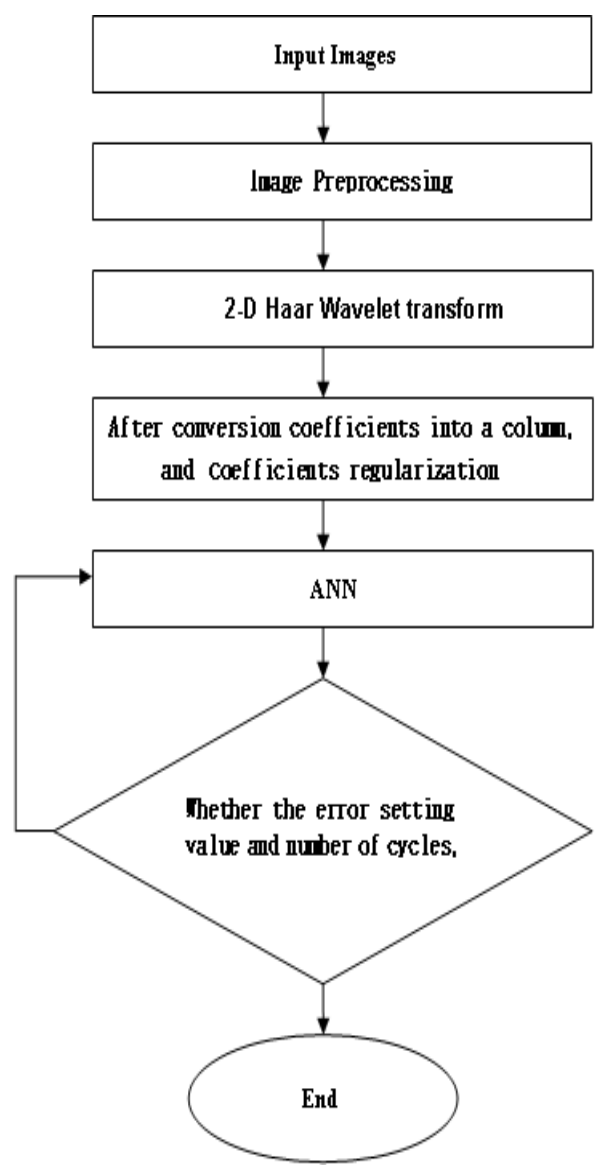

Fig. 2. License plate training flow chart. 


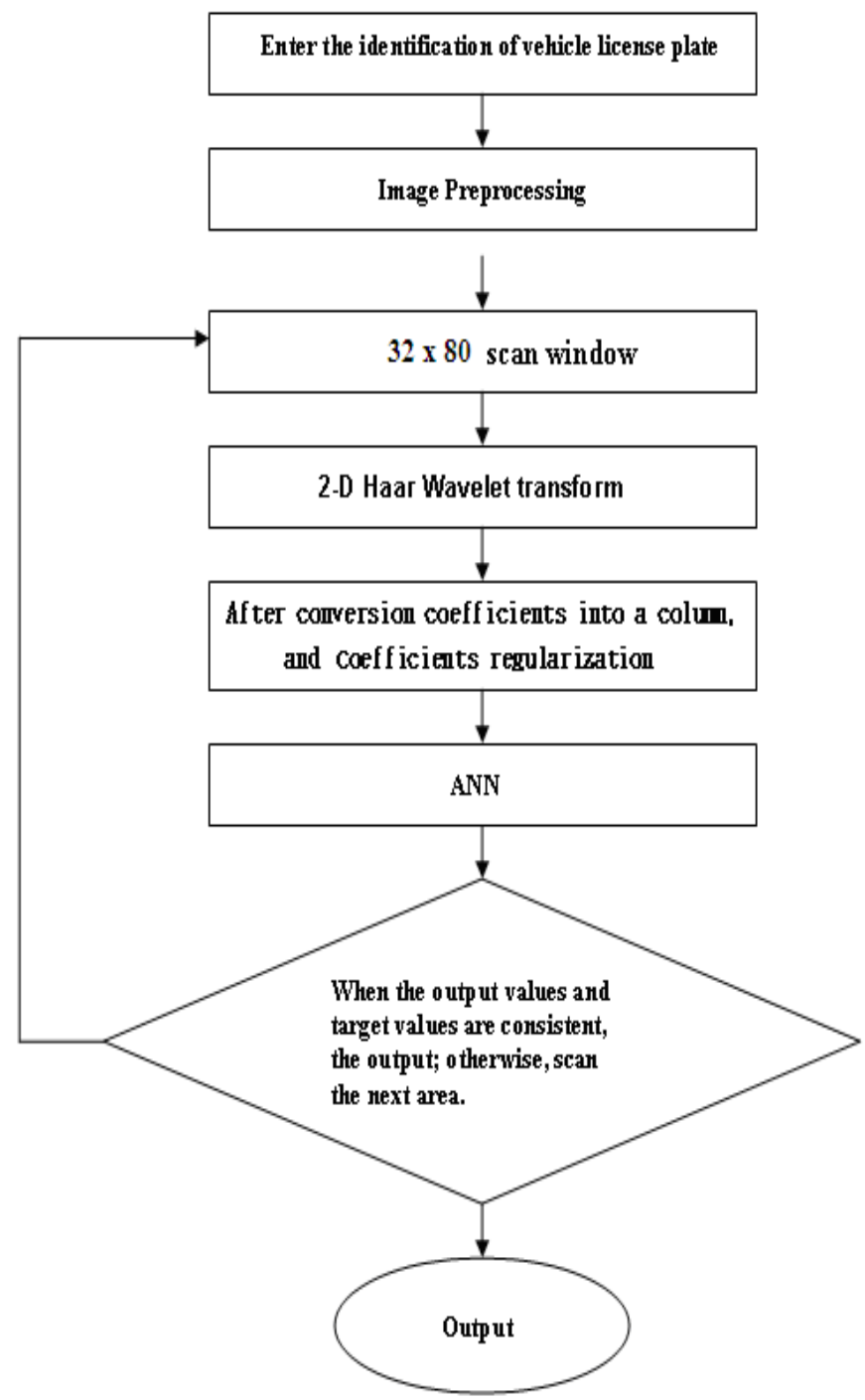

Fig. 3. License plate recognition flow chart.

\subsection{License Plate Training Steps Process}

In this article, license plate training processes have seven steps, as show in Fig. 2, described it as follows.

Step1. Enter vehicle license plate images, as in Fig. 4(a), Fig. 6(a), Fig. 8(a) and Fig. 10(a).

Step2. Vehicles license plates image preprocessing (as Fig. 4, Fig. 6, Fig. 8 and Fig. 10).

2.1). The color photo will use (12) equation to transform the gray scale image, $Y$ is gray level image after image the transformation, $\mathrm{R}, \mathrm{G}$ and $\mathrm{B}$ is the primitive color image element. Because hitting the inductive loop vehicle and location of fixed, image preprocessing to reduce the top 150 pixels, and under the most, after cutting 100 pixels to the right and left, cutting away does not affect the license plate displayed, and this can reduce background noise, and running time.

$$
Y(i, j)=(R(i, j)+G(i, j)+B(i, j)) / 3
$$

$Y(i, j)$ are gray-scale space red, green and blue color components.

2.2). Vehicle images utilized edge function and Sobel mask operator for license plate localization, it can automatically detect the critical threshold, the following (3) formula is MATLAB software program. Vehicle images transform into 0 and 1 binary, making license plate and background distinct, as show in Fig. 5, Fig. 7, Fig. 9, and Fig. 11. 
2.3). Using license plate district, height, width, width to height ratio and distance up and down the second one taking a line to extract license plates, as show in Fig. 12, Fig. 13, Fig. 14, and Fig. 15.

Step3. License plate Haar Discrete Wavelet Transformation two times, after transform only selected LL low-frequency parts, as show in Fig. 16, Fig. 17, Fig. 18, and Fig. 19.

Step4. LL the low frequency part of the coefficients arrange into a column as license plate the feature values, and then utilize (13) formula regularization when the Artificial Neural Network (ANN) input data.

$$
\frac{X_{i}}{\max \left(X_{i}\right)}
$$

$X_{i}$ : License plate coefficients of the feature vector.

Step5.Use Artificial Neural Network to train. Set ANN parameter, neurons number of input layer is the license plate feature value numbers, neurons number of hidden layer is (input layer of neurons + output layer neurons)/2, number neurons of output layer is 8 , and train cycle is 1000 , learning rate 0.5 , and the four initial weight and basis values are randomly generated by a computer program.

Step6.Discriminant. (If the up to train cycle and convergence stopped, otherwise continue to train. After successful training, save weight and basis values )

Step7. End.

\subsection{License Plate Recognition Steps Process}

These are eight recognition steps described in below:

Step1. Enter vehicle license plate images, such as Fig. 4(a), Fig. 6(a), Fig. 8(a), and Fig. 10(a). Such as Fig. 1 of 12-0 symbols of the region, in different locations of Photo 6, 100 vehicles, a total of 600, photos taken when the recognition of samples.

Step2. Vehicles license plates image preprocessing (as Fig. 4, Fig. 6, Fig. 8, and Fig. 10).

Step3. A $32 \times 80$ scan window to scan the image, and at the same time through Haar Discrete Wavelet Transform two times of the image scan area, as show in Fig. 16, Fig. 17, Fig. 18, and Fig. 19.

Step4. After step3, select only the second transformation of LL low-frequency parts, the image becomes $8 \times 20$ size.

Step5. The $8 \times 20$ pixels arrange into a column, as each scan the regional feature values. And then the feature values of regularization, as such ANN input data.

Step6. Use Artificial Neural Network to recognize. (Set input neuron is the feature values number, the hidden neurons is 84 , and the output layer neurons is 8 , and load the train obtain weight and basis values.)

Step7. Discriminant. (If you enter a value of can satisfy the class of ANN training is complete weight and basis values, the recognition was successful, otherwise continue to scan an image next region.)

Step8. Output the recognition results.

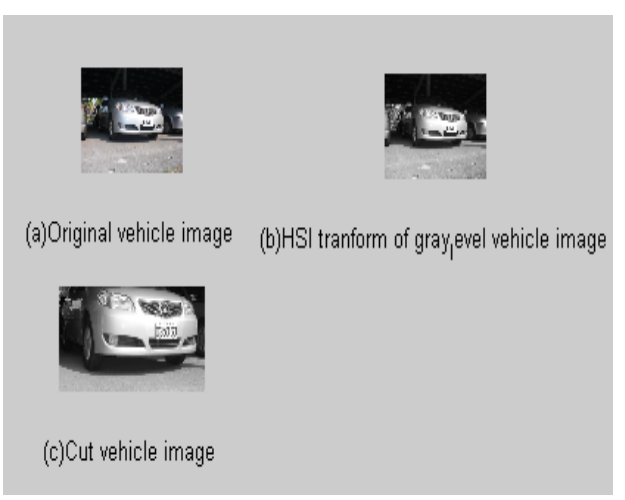

Fig. 4. Daytime image preprocessing.

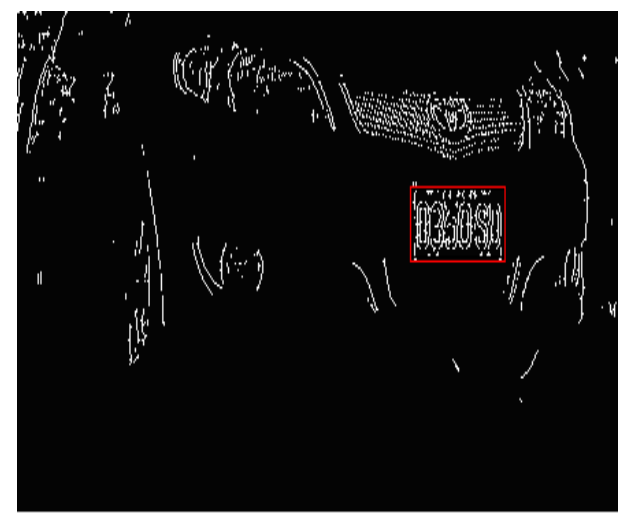

Fig. 5. Daytime edge of vehicle image. 


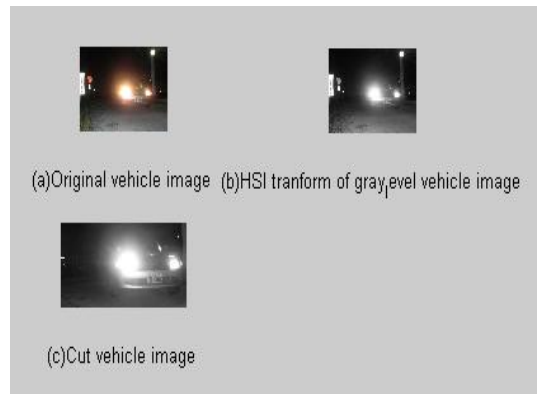

Fig. 6. At night image preprocessing.

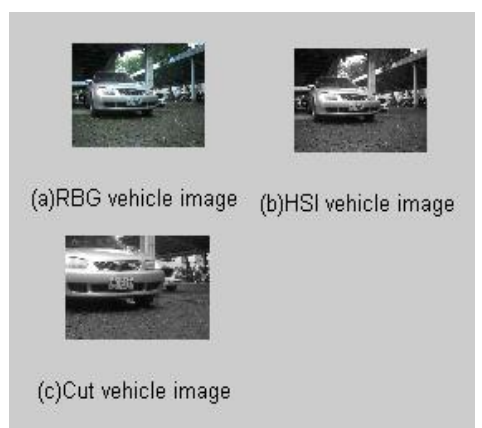

Fig. 8. Little rain day image preprocessing.

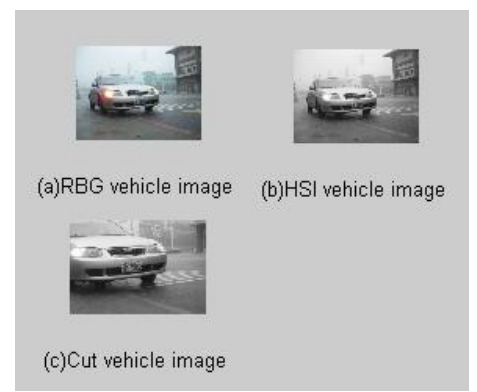

Fig. 10. Rain day image preprocessing.

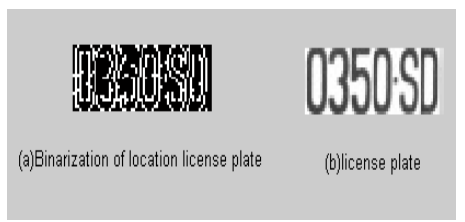

Fig. 12. (a) Daytime license-plate location.

(b) License plate.

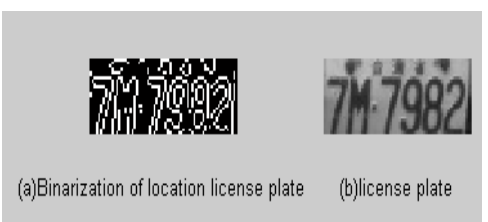

Fig. 14. (a) Little rain day license-plate location.

(b) License plate.

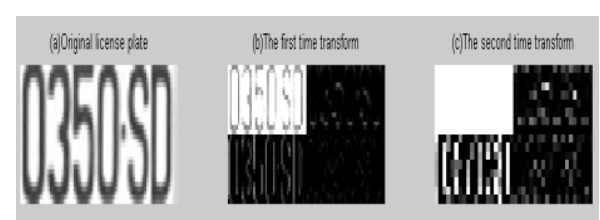

Fig.16. 0350-SD license plate HDWT two times transform.

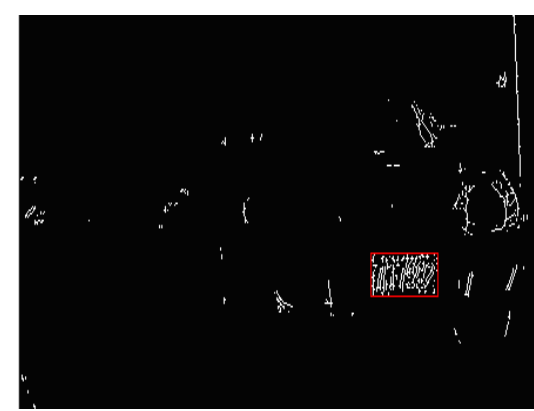

Fig. 7. At night edge of vehicle image.

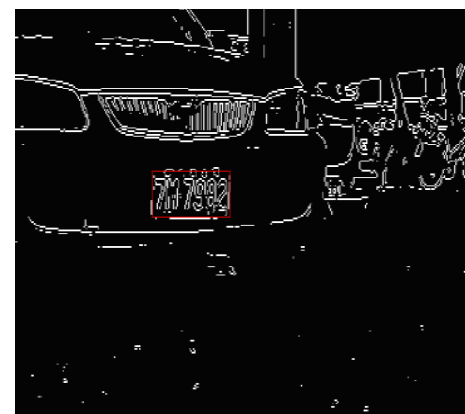

Fig. 9. Little rain day edge of vehicle image.

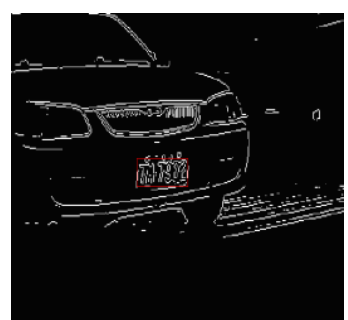

Fig. 11. Rain day edge of vehicle image.

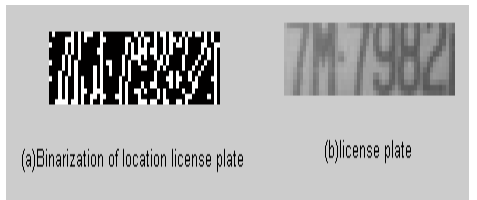

Fig. 13. (a) At night license-plate location. (b) License plate.

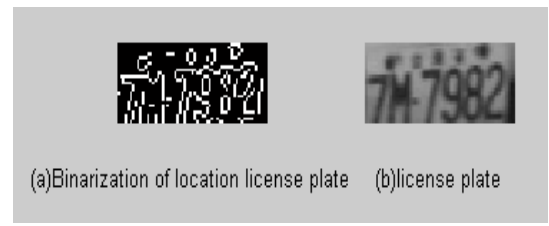

Fig. 15. (a) Rain license-plate location. (b) License plate.

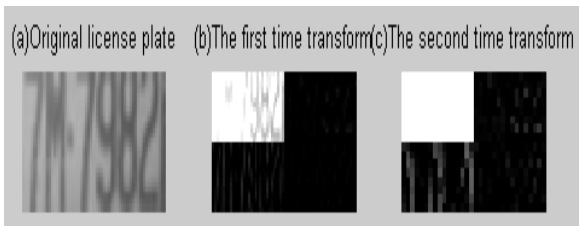

Fig.17. 7M-7982 license plate HDWT two times transform. 


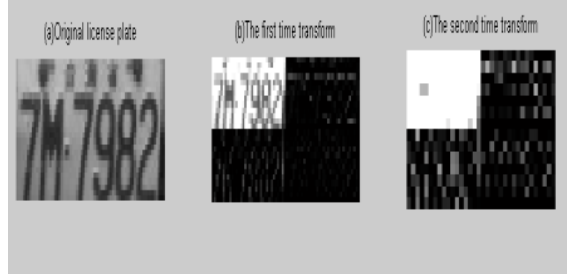

Fig.18. Little rain day 7M-7982 license plate HDWT two times transform.

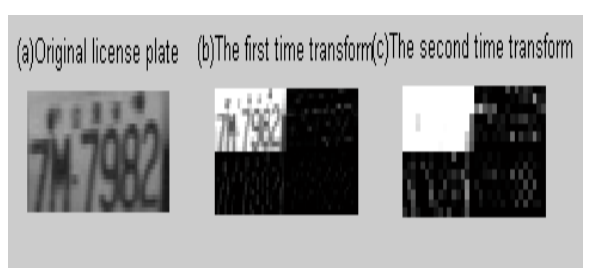

Fig. 19. Rain day 7M-7982 license plate HDWT two times transform.

\section{Experimental Result and Analysis}

In Fig. 1 the 12 of 0 sign locations photo, 100 vehicles, a total of 1200 sheets vehicle license plate for train, and in Fig. 1 the 12 locations within the region, each vehicle license plate photo taken 6 pictures, 100 vehicles, a total of 600 sheets as recognition data. We are in the different location to photograph and different sizes license plate, collected the image displays the following properties: (1) From the left lateral light photography with more complex backgrounds. (2) And some pictures as the evening sunlight and low quality. (3) According to the distance was taken different plate sizes vary.

\subsection{License Plate Recognition Results}

License plate recognition results as from Figs. 20 to 23 shows.

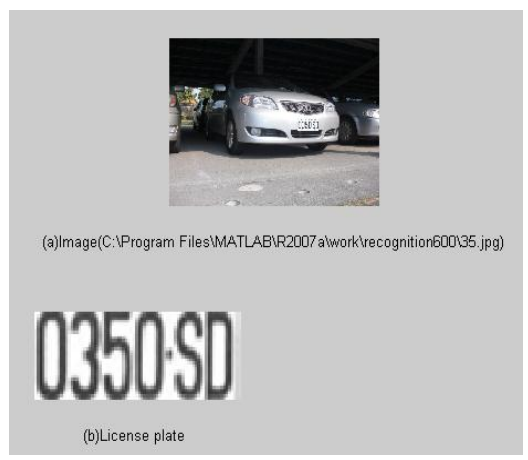

Fig. 20. At daytime recognition result.

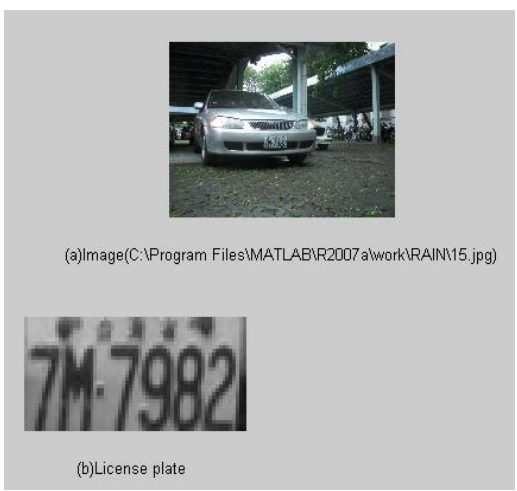

Fig. 22. Little rain day recognition result.

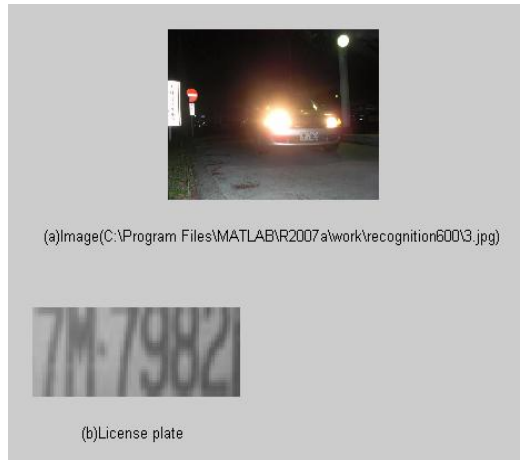

Fig. 21. At night recognition result.

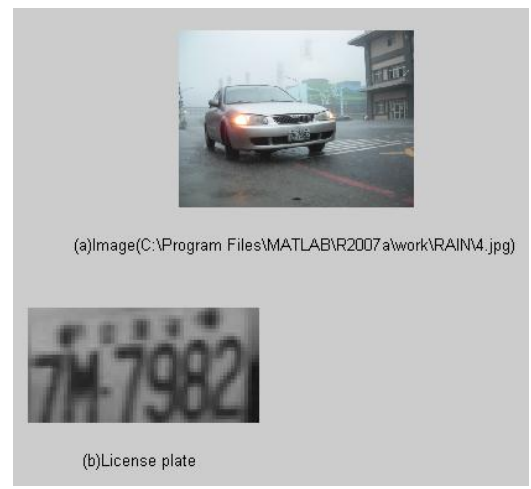

Fig. 23. Large rain day recognition result.

\subsection{Recognition and Analysis of Results}

This simulation within limited areas and a limited number of vehicle recognition plates. Photo license plate recognition is in mixing and good weather conditions on the day and night. The vehicle license plate recognition system, the first step is to extract license plate by ANN training, train complete obtained weight and basis values. The second step is to use the $32 \times 80$ scan window, scan unknown vehicle license plate, and load ANN training completed of weights and basis values, and license plate recognition with Artificial Neural Network. If it recognizes successfully, opening the door for a vehicle to enter. If it recognized failed, forbids the vehicle to enter. 
This experiment in license plate location and vehicle license plate extraction, In 1200 vehicles license plate image have 76 license plates location and extraction failed, accurate rates is approximately 93.67\%, failed due to car light, strong sunlight, background around the vehicle and license plate-like parts of a complex background, and so on. In recognition of 600 vehicles photo have 29 failed, recognition rates of approximately $95.17 \%$. This article, and references [8], [19], [21] recognition rate compare with, show in Table 1.

Table1. This article compare with references [8], [19], [21].

\begin{tabular}{ccc}
\hline Paper No. & Recognition rate (\%) & sort number \\
\hline This article & 95.17 & 1 \\
{$[19]$} & 94.7 & 2 \\
{$[21]$} & 94.5 & 3 \\
{$[8]$} & 94.2 & 4 \\
\hline
\end{tabular}

\section{Conclusion}

This papers by made of license plate recognition system is different from traditional of license plate recognition system, its characteristics is directly scan and the using 2-D Haar discrete wavelet two times conversion, and every time conversion image take low frequency part, makes image became original image of $1 / 4$ coefficient, makes run time became $1 / 4$ times time, and by two times conversion image extract low frequency part LL of vehicles license plate image, makes image coefficient became original of one-sixteen, Thus reducing coefficient recognition plate imaging, fast recognition speed.

This paper recognition system is a combination of 2-D Haar discrete wavelet transform and artificial neural networks are license plates through the 2-D Haar discrete wavelet transform, get the license plate of the feature values, and characteristic values into a column, then the feature values corresponding to license plate type artificial neural network training, when training is complete, using training received loads of weight values and weights of artificial neural networks. Then, using $32 \times 80$ scan window progressive scan unknown effects of vehicle license plate recognition, and outputs the results. MATLAB software test result, license plate recognition rate up to $95.17 \%$.

\section{References}

[1] Sheng, H., Li, C., Wen, Q., \& Xiong, Z. (2009). Real-time anti-interference location of vehicle license plates using high-definition video. IEEE Intelligent Transportation Systems Magazine.

[2] Wijetunge, A. W. G. C. D., \& Ratnaweera, D. A. A. C. (2011). Real-time recognition of license plates of moving vehicles in Sri Lanka. Proceedings of the 2011 6th International Conference on Industrial and Information Systems.

[3] Hai, L., \& Shrestha, G. (2010). Real time license plate recognition at the time of red light violation. Proceedings of the International Conference on Computer Application and System Modeling.

[4] Conci, A., Carvalho, J. E. R. D., \& Rauber, T. W. (2009). A complete system for vehicle plate localization, segmentation and recognition in real life scene. IEEE Latin America Transactions, 7(5).

[5] Wei, X. (2010). Seeds algorithm in vehicle license plate location system. Proceedings of the Second IITA International Conference on Geoscience and Remote Sensing.

[6] Wu, M. K., Wei, L. S., Shih, H. C. \& Ho, C. C. (2009). License plate detection based on 2-level 2-D Haar wavelet transform and edge density verification. Embedded SoC Lab, Department of Electrical Engineering, National Yunlin University of Science and Technology, Seoul, Korea.

[7] Song, Y. (2010). A level set based method of license plate localization. Key Laboratory of Intelligent Information Processing, Institute of Computing Technology, Chinese Academy of Sciences, IEEE IRI 2010, 
Las Vegas, Nevada, USA, 403-404.

[8] Kulkarni, P., Khatri, A., Banga, P., \& Shah, K. (2009). A feature based approach for localization of Indian number plates. Dept. of Electronics and elecommunication, Smt. Kashibai Navale College of Engineering, University of Pune, India, IEEE, 157-162.

[9] Majid, P. M. P., Yaacob, M. S. A. l. S., Rahiman, M. H. F., \& Krishnan, R. P. (2009). Statistical time energy based damage detection in steel plates using artificial neural networks. International Colloquium on Signal Processing and Its Applications.

[10] Chen, N., \& Xing, L. (2010). Research of license plate recognition based on improved BP artificial neural network. Proceedings of the International Conference on Computer Application and System Modeling.

[11] Ko, M. A., \& Kim. Y. M. (2003). License plate surveillance system using weighted template matching. Proceedings of the 32nd Applied Imagery Pattern Recognition Workshop.

[12] Shan. B. (2011). Vehicle license plate recognition based on text-line construction and multilevel RBF neural network. Journal of Computers, 6(2), 246 - 253.

[13] Abdullah, S. N. H. S., Khalid, M., \& Yusof, R. (2006). License plate recognition using multi-cluster and multilayer neural networks. IEEE, 1818 - 1823.

[14] Porikli, F., \& Kocak, T. (2006). Robust license plate detection using covariance descriptor in a neural network framework. Proceedings of the IEEE International Conference on Video and Signal Based Surveillance.

[15] Rahman, C. A., Badawy, W., \& Radmanesh, A. A real time vehicle's license plate recognition system. Proceedings of the IEEE Conference on Advanced Video and Signal Based Surveillance.

[16] Faradji, F., Rezaie, A. H., \& Ziaratban, M. (2007). A morphological-based license plate location. ICIP2007.

[17] Crovato, C. D. P., \& Schuck, A. (2007). The use of wavelet packet transform and artificial neural networks in analysis and classification of dysphonic voices. IEEE Transactions on Biomedical Engineering, 54(10), 898 - 1990.

[18] Anagnostopoulos, C., Anagnostopoulos, I., Kouzas, G., Loumos, V., Kayafas, E., \& Tsekouras, G. (2005). Using sliding concentric windows for license plate segmentation and processing. IEEE(pp. 337-342).

[19] Ronghui, F. (2016). The research and design of vehicle license plate recognition system in traffic management system. International Journal of Signal Processing, Image Processing and Pattern Recognition, 9(3), 445 - 456.

[20] Anuj, K. (2016). Character recognition in automatic vehicle license plate recognition. International Journal of Advanced Research in Computer Science and Software Engineering, 6(7), 393 - 396.

[21] Arulogun, O. T., \& Amusan, D. G. (2013). Vehicle license plate recognition using edge detection and neural network. International Journal of Science and Researc.

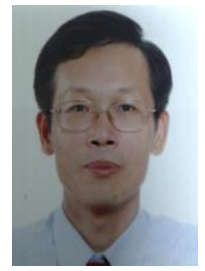

Lee Rong-Choi was born in Taiwan, Republic of China, on 20,1961, He is a PhD student at National Kaohsiung First University of Science and Technology, Computer and Communication Engineering. His research interests are image processing, network communication, message encoding, and engaged in electrical work. He had published many articles in journal and IS3C2012 conference.

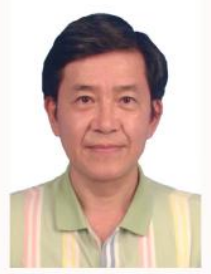

King-Chu is a professor of the Republic of China Kaohsiung First University of Science and Technology, His main research is image processing and signal processing, and breast cancer to identify and ECG symptoms to identify. 